Article

\title{
Using the Hall Effect for Monitoring the Starter Condition in Motor Vehicles
}

\author{
Mieczysław Dziubiński ${ }^{1, *}$, Grzegorz Litak ${ }^{2,3, *}$, Artur Drozd ${ }^{4}$, Kamil Szydło ${ }^{1}$, Rafał Longwic ${ }^{1}$ \\ and Piotr Wolszczak 2 (iD \\ 1 Department of Automotive Vehicles, Lublin University of Technology, Nadbystrzycka 36, 20-618 Lublin, \\ Poland; kamil-szydlo@wp.pl (K.S.); r.longwic@pollub.pl (R.L.) \\ 2 Department of Automation, Lublin University of Technology, Nadbystrzycka 36, 20-618 Lublin, Poland; \\ p.wolszczak@pollub.pl \\ 3 Department of Process Control, AGH University of Science and Technology, Mickiewicza 30, \\ 30-059 Kraków, Poland \\ 4 Department of Thermodynamics, Fluid Mechanics and Aviation Propulsion Systems, \\ Lublin University of Technology, Nadbystrzycka 36, 20-618 Lublin, Poland; adrozd.d@gmail.com \\ * Correspondence: m.dziubinski@pollub.pl (M.D.); g.litak@pollub.pl (G.L.)
}

Received: 13 March 2018; Accepted: 2 May 2018; Published: 9 May 2018

\begin{abstract}
We present a new method of controlling the operational wear of the starter used for transport. The new method consists in making use of the magnetic field in the armature gap as a diagnostic signal for determining fitness for use. Testing the starter with the Hall sensor involved recording the voltage course obtained from the sensor, which was commensurate with magnetic induction. The tests were carried for the unloaded starter, i.e., while running idle, as well as for the loaded one. As part of the experiment, simulation of different kinds of operational wear and selected simulated failures of starter elements were carried out. The objective of this study is to elaborate a diagnostic model for the operational control of the starting system for motor transport.
\end{abstract}

Keywords: Hall effect; starter; diagnostics; measurements; control parameters

\section{Introduction}

Two important factors in the process of using a car are its reliability and running costs. They depend not only on the vehicle structure but also on the assumed operational rules. The handling system serves the purpose mainly of restoring (regenerating) the running potential of the vehicle, which decreases as a result of natural wear or damage caused by wrong operation or errors and faults occurring in the process of construction or production.

The introduction of ever better methods of diagnostic tests makes it easier to use the method of restoring the running potential, which consists in repairing or replacing particular systems before they reach their boundary state, and most of all before a failure occurs, making a vehicle unfit for use [1-4]. Functional diagnostics conducted on a regular basis ensures reliable work of the starter, its optimal usage, and in particular the prevention of unexpected failures in the course of vehicle operation [5-8]. In order to achieve the basic goals of functional diagnostics, it is important to choose such starter parameters, which can optimally allow one to draw conclusions about its technical condition. Diagnostics can predict in advance when the starter's basic elements approach their boundary or critical condition [9-12].

The aim of this study is to work out a new method of controlling the operating wear of the car starter. The method consists in making use of a magnetic field in the armature slot as a diagnostic signal for determining fitness for use. To achieve the goal of discovering a method of starter functional 
diagnostics, a test stand was designed and constructed, and the test objects (i.e., starters) were properly prepared.

\section{Analysis of the Magnetic Circuit and Distribution}

The magnetic circuit of the starter can be divided into two basic parts:

- $\quad$ stator-a stationary part;

- $\quad$ rotor-a movable part.

The two parts are separated by an air gap called the armature gap. In the stator part of the magnetic circuit, the following elements can be distinguished: a pole shoe, a main pole core (pole body), an air gap between a core and a yoke (when a core and a yoke are made of separate parts), and a yoke [13].

In the rotor part, the elements of the magnetic circuit are the following: armature teeth and the armature core. The size of the armature gap has a significant impact on the characteristics and dimensions of the machine. On the one hand, it is advisable to make the size of the $\delta$ gap smaller because it causes the decrease in the magnetic tension of the gap. At the same time, the dimensions of the machine may become smaller because less space is necessary to accommodate excitation winding. This makes it possible to decrease the height of the pole and consequently to make the outer diameter of the machine smaller at a constant armature diameter. Excitation losses are becoming smaller as well. However, at small values of $\delta$, the magnetic field of the machine changes when there are load variations, which has a negative impact on commutation and leads to its unstable work [14]. The size of the armature gap is usually not constant along the armature perimeter because the arch of the pole shoe may be of different shapes depending on the required distribution of magnetic field in the gap. Most frequently, the gap is constant and concentric along the rotor (about $0.6 \div 0.7$ of the pole piece width), and, near the pole horns, it increases to $2 \delta$.

If the gap was the same along the whole arch of the pole, a rapid change in magnetic induction on the pole horns would occur. It would cause more losses in teeth steel and in armature winding and the increase of noise generated by the machine [15-17].

The properties of electric machines depend on the distribution of the radial constituent $B_{r}$ of magnetic induction in the air gap between the stator and the rotor [18]. The course of function $B_{r}=\mathrm{f}(\alpha)$, describing the distribution of induction depending on angle $\alpha$, is called the field distribution or the magnetic field curve (Figure 1).

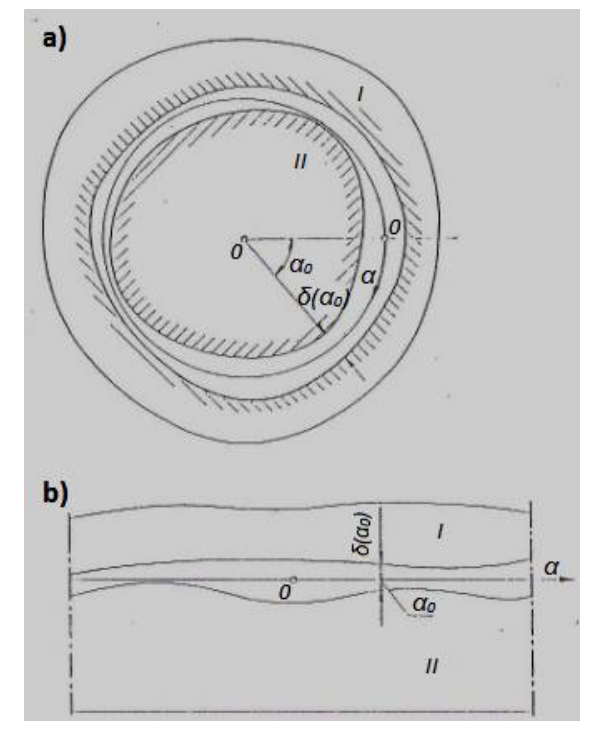

Figure 1. The cross section of Stator I and Rotor II cores: (a) cylindrical layout; (b) equivalent flat layout. 
Field distribution depends on the following factors [19]:

1. the current distribution along the inner circuit of the stator core and the outer circuit of the rotor core,

2. the distribution of magnetization vector of permanent magnets,

3. the shape of the air gap between the stator and the rotor, and

4. the magnetic tension in stator and rotor cores.

Even at drastic simplifications consisting in neglecting magnetic tension in the core and assuming smooth by-gap surfaces of the armature and the poles, it is difficult to formulate analytical dependency describing induction distribution in the gap depending on the dimensions of the magnetic circuit and the winding. Such difficulties are the result of the complex shape of magnetic circuit elements. In the analysis of induction distribution in the electric machine, there are many additional simplifications.

It is assumed that the magnetic field in the gap is flat, i.e., the winding wires are parallel to the rotor rotation axis; in the core there are no radial passages; the deformation of field distribution on the core edges is neglected [20]. The gap length in radial direction is so small in comparison with the other core dimensions that, for the purpose of analyzing field distribution, the cylindrical layout can be replaced with the flat one after outspreading the core which has been cut open (Figure 1b).

\subsection{Magnetic Field from Excitation Winding}

Induction in gap $B_{\delta f x}$ from excitation winding is proportional to field strength $H_{\delta f x}$.

$$
B_{\delta f x}=\mu_{s} \times H_{\delta f x}
$$

where air magnetic permeability $\mu_{s}=4 \pi \times 10^{-7} \mathrm{H} / \mathrm{m}$.

The strength of magnetic field $H_{\delta f x}$ can be expressed by the following formula when magnetic tension drops in the iron core are neglected:

$$
H_{\delta f x}=\frac{\mu_{\delta f x}}{l_{\delta f}}
$$

When magnetic tension drops in the air gap,

$$
U_{\delta f x}=F_{f}=0.5 \Theta_{f}
$$

where $F_{f}$ is the magnetic tension of magnets, and $\Theta_{f}$ is the excitation flow of one pair of poles.

$$
\Theta_{f}=\frac{z_{f}}{p} I_{f}
$$

where $z_{f}$ is the number of coils on all poles, $p$ is the number of pole pairs, and $I_{f}$ is the excitation current.

The gap length is calculated as follows:

$$
l_{s x}=k_{c} \times \delta(x) .
$$

The Carter coefficient $k_{c}$ denotes a calculative increase of the air gap as a result of the existence of grooves, usually $k_{c}=1.05 \div 1.2$. Eventually, the distribution of induction from excitation winding can be approximately determined from the following formula:

$$
B_{\delta f x}=\frac{\mu z_{f}}{2 p k_{c} \delta(x)} .
$$

Function $\delta(x)$, present in the formula, is determined on the basis of the set shape of the pole shoe. The interpolar gap and the edges of the pole shoe are the places where equivalent gap length 
equal to the length of magnetic field lines between the armature and the pole can be determined. Field distribution is determined separately for each pole, assuming that there are no adjacent poles. Then, by summing field distributions of two consecutive poles, the resultant distribution can be obtained from the formula:

$$
B_{\delta f x}=B_{N}(x)+B_{S}(x) .
$$

An example of induction distribution for a machine with a concentric gap is presented in Figure 2, in which the pole pitch

$$
\tau=\frac{\pi D}{2 p}
$$

where $D$ is the armature diameter, and $p$ is the number of pole pairs.

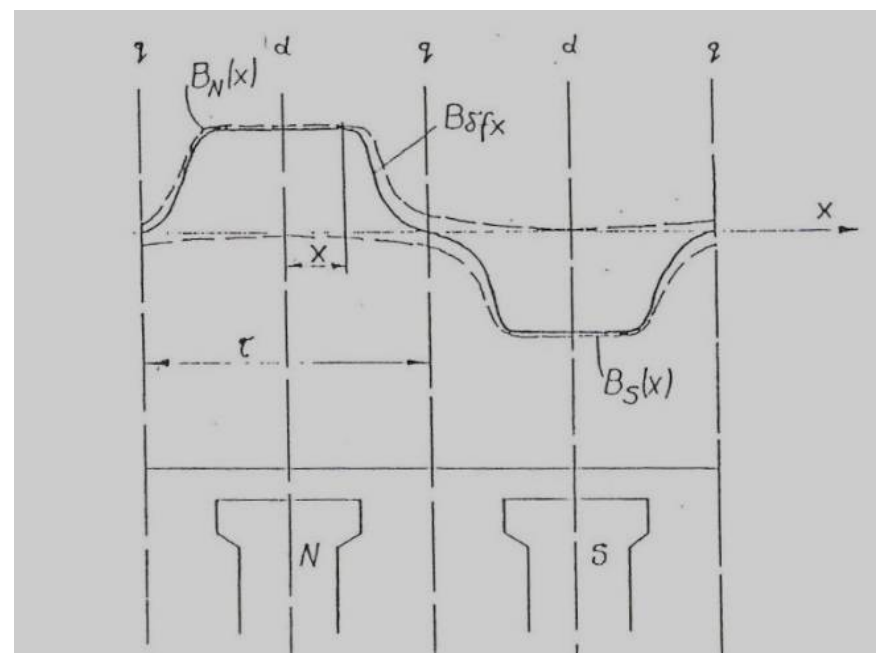

Figure 2. Distribution of magnetic induction from excitation winding on the outspread circuit of the machine with a commutator gap.

\subsection{Magnetic Field from Armature Current}

A machine with current $I_{a}$ flowing in bars laid in grooves can be treated as a machine with a layer of current placed on the machine circuit, i.e., with a loading of current A. The loading of current from the armature of the direct current machine can be determined by Equation (9):

$$
A=\frac{N I_{g}}{\pi D}=\frac{N I_{a}}{2 a \pi D}=\frac{N I_{a}}{4 a p \tau}
$$

where $I_{g}$ is the current in particular branches, $I_{a}$ is the current in the circuit of brushes, $a$ is the number of parallel branch pairs, $p$ is the number of pole pairs, and $\tau$ is the pole pitch

When the brush axes are placed in axis $q$, the flow direction $\Theta_{a}$ is equal to the direction of axis $q$. When the armature moves, the flow direction $\Theta_{a}$ does not change. If magnetic tension drops in iron cores are neglected, magnetic tension in the gap (between the surface of the armature and the surface of the pole shoe) in point $\mathrm{c}$ is determined by the following formula:

$$
U_{\mu \delta a x}=\frac{1}{2} \Theta_{2 x}=A x
$$

and is shown as a straight line $U_{\mu \delta a x}$ (Figure 3). 


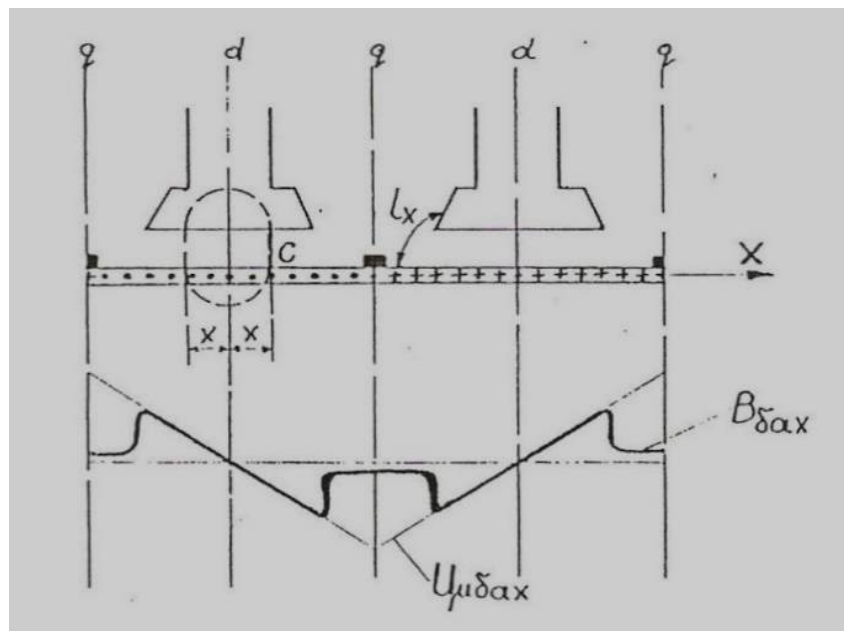

Figure 3. Magnetic induction $B_{\delta \alpha x}$ from armature current when the brushes are placed in axis $q$.

Magnetic field strength is expressed as follows:

$$
H_{\delta a x}=\frac{U_{\mu \delta a x}}{l_{x}}=\frac{A}{l_{x}} x
$$

where $l_{x}$ is the length of flow track in the air, $\left(l_{x}=k_{c} \cdot \delta(x)\right)$

Magnetic induction in the gap from armature current is expressed as follows:

$$
B_{\delta a x}=\mu_{\delta} \times H_{\delta a x}=\frac{\mu_{\delta} A}{k_{c} \delta(x)} x .
$$

Figure 3 presents curve $B_{\delta a x}$, which illustrates induction distribution $B_{\delta a x}$ for brushes placed in axis $q$.

\subsection{Resultant Magnetic Field of the Starter}

In the direct current engine, resultant induction in gap $B_{\delta x}$ is the sum of induction in the gap from excitation current $B_{\delta f x}$ and induction in the gap from the armature current (from armature reaction) $B_{\delta a x}$.

$$
B_{\delta x}=B_{\delta f x}+B_{\delta a x}
$$

The resultant induction is smaller under half of the pole piece as a result of magnetic circuit saturation. Figure 4 presents the distribution of the resultant induction.

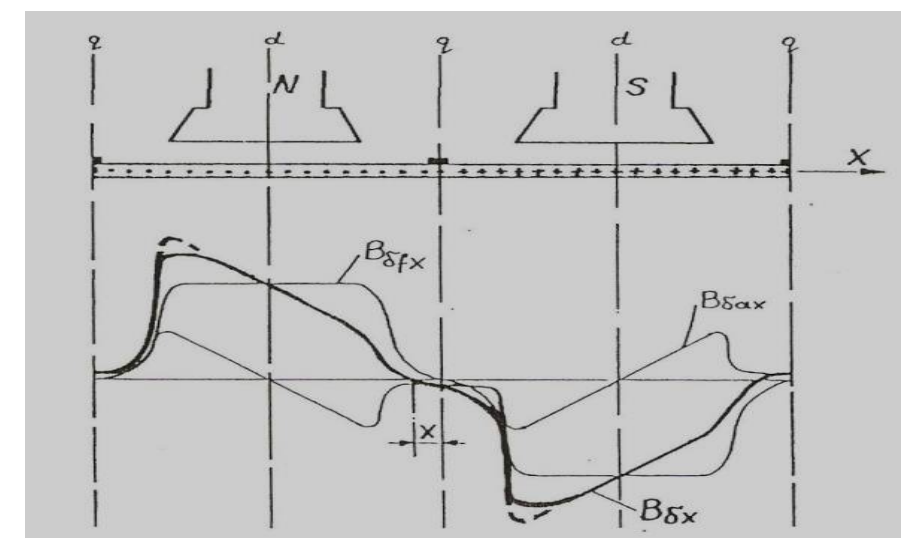

Figure 4. Resultant magnetic field of the direct current engine with brushes placed in axis $q$. 
By simulating the operational wear of the starter sleeve that changes the armature gap, the distribution of the magnetic flux and the magnetic induction in the gap were determined. Figure $5 \mathrm{a}$ shows the spread of the magnetic flux for the starter R5 for the gap in a segment of the commutator. Figure $5 \mathrm{~b}$ shows the distribution of the induction for an armature gap of $1 \mathrm{~mm}$, as well as the damage in the form of the gap in two segments of the commutator and the worn sleeve of the starter R5. Figure $5 \mathrm{c}$ shows the distribution of the induction for the efficient starter. The new diagnostic method allows one to clearly identify the degree of the operational wear of the sleeves as well as the damage in the form of interruptions and short-circuits of the rotor windings. The simulation was performed on the real object using the QuickField program. Apart from starter dimensions, data on the type of excitation, the number of starter grooves, and the material magnetization curves for the rotor and stator were introduced to the program. A variable value of the armature gap was introduced, resulting from the wear of the sleeve. The simulation results indicate that the highest values of magnetic induction occur in the four sections of the yoke between the pole shoes and the yoke gap. For an efficient starter (Figure 5c), the maximum induction value is $0.8 \mathrm{~T}$.

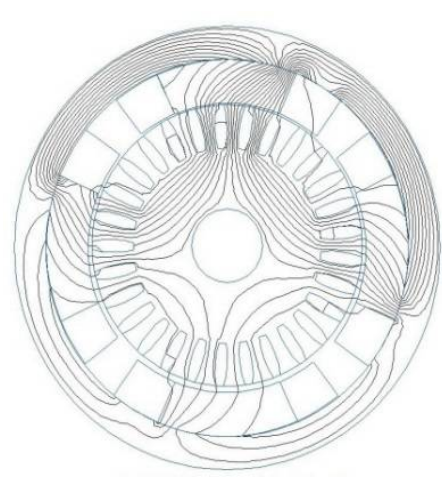

(a)

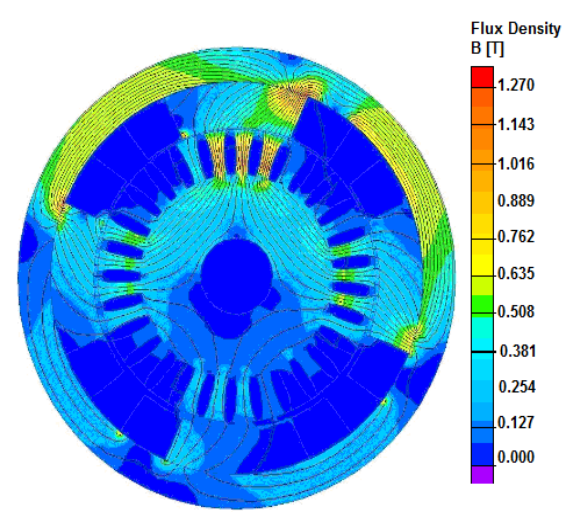

(b)

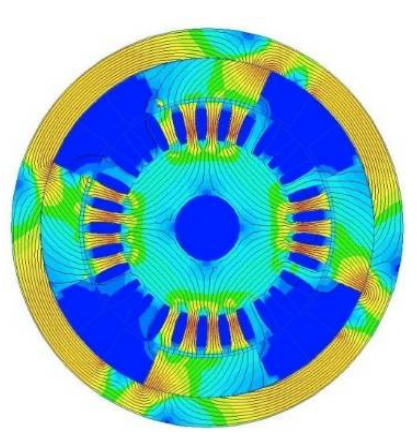

(c)

Figure 5. The starter R5 spread of magnetic flux for the gap in a segment of the commutator (a); the distribution of magnetic induction for the gap in two segments of the commutator and the worn sleeve (b) and efficient starter (c).

\section{Experimental Studies of Induction Distribution in the Magnetic Gap}

The starter R76a with two Hall sensors (DKWP 10-40) was used in tests. One of them was placed on the rotor, the other one on the stator. The tests were conducted for two load states, i.e., idle running and partial load. The simulation of failures was limited to chosen cases:

(1) rotor winding ground;

(2) the shorting of two commutator sectors;

(3) the breaking of the integrity of rotor winding;

(4) the shorting of an excitation winding coil;

(5) wear of brushes.

The simulation was limited because certain failures, such as the stator winding ground made it impossible for the starter to work, even when it was idling.

\subsection{The Description of the Test Stand}

\subsubsection{The Object Tested}

The starter R76 is a series direct current motor adjusted for working in a one-conductor wiring system with a voltage of $12 \mathrm{~V}$. It is equipped with an electromagnetic switch closing the main circuit 
and a coupling mechanism triggering the mesh of the starter coupling system with the engine flywheel for the time of the start-up. The coupling system placed on a screw spline is equipped with a four-roller unidirectional clutch, which protects the rotor of the starter from damage caused by external drive. The friction system made of fiber pads makes the rotor brake the very moment the power supply is cut off, which enables one to connect the rotor again very quickly. The starter is intended to start a spark ignition engine.

\subsubsection{Connecting and Fixing Hall Sensors}

The Hall sensor is fixed in pole shoe milling and the wires are introduced through openings in the pole piece and the frame. Figure $6 \mathrm{a}$ shows how the wires are connected, and Figure $6 \mathrm{~b}$ shows the Hall sensor placed on the pole shoe in the starter. Figure 7 shows the connection of the Hall sensors, and Figure 8a presents the view of the starter. The Hall sensor on the rotor is shown in Figure 8b.

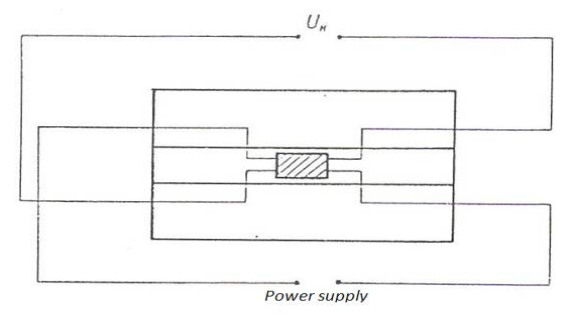

Pinion

(a)

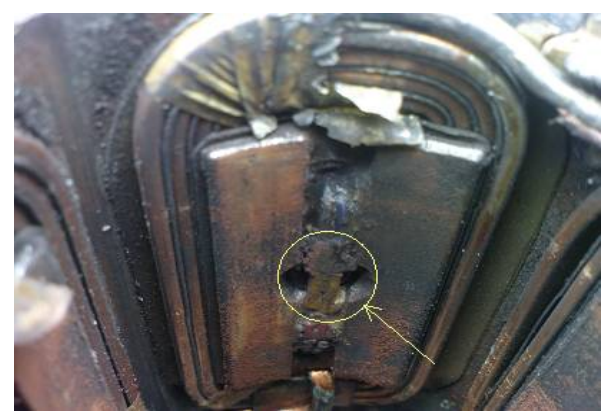

(b)

Figure 6. Connections of the Hall sensor placed on the pole shoe (a) and the Hall sensor placed on the pole shoe in the starter $(\mathbf{b})$.

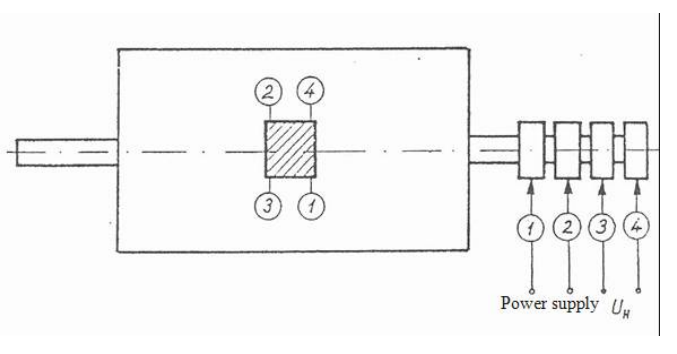

Figure 7. Connections of the Hall sensor placed on the rotor.

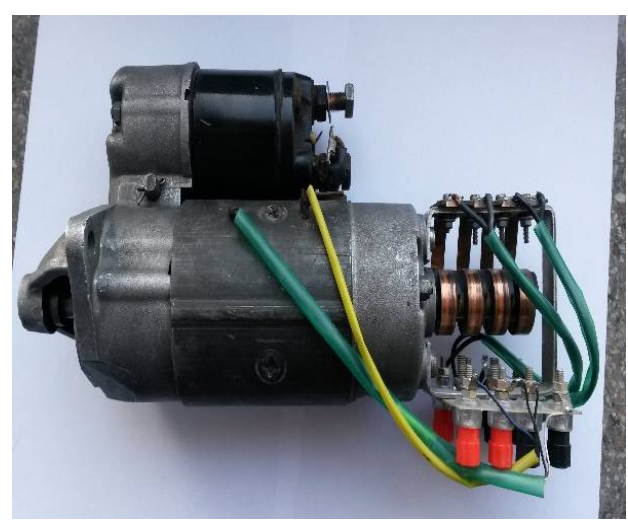

(a)

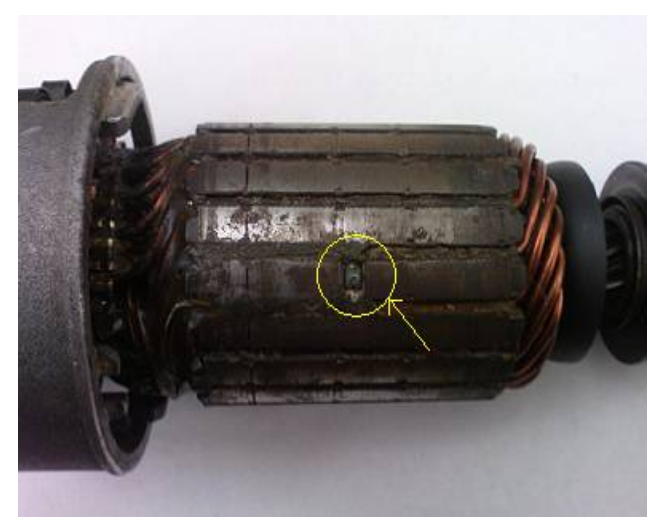

(b)

Figure 8. (a) View of the starter with fitted Hall sensors (b) and the Hall sensor placed on the rotor. 
The Hall sensor is stuck in the rotor tooth notch halfway through its length. Electric cables laid in the rotor grooves and in the rotor axis hollow are connected to four slip rings placed on the rotor axis, specially lengthened for this purpose.

\subsubsection{Measurement Stand}

In the course of tests, the starter was mounted on an Elkon Super 3 type test stand. Such a stand was used only for loading the starter with a constant moment value. The starter was supplied by a separate $12 \mathrm{~V}$ battery. The signal from the Hall sensor was passed to the amplifier and from the amplifier through the protective system to the computer with an analogue-to-digital converter. Figure 9 presents the diagram of the stand. The view of the stand is shown in Figure 10.

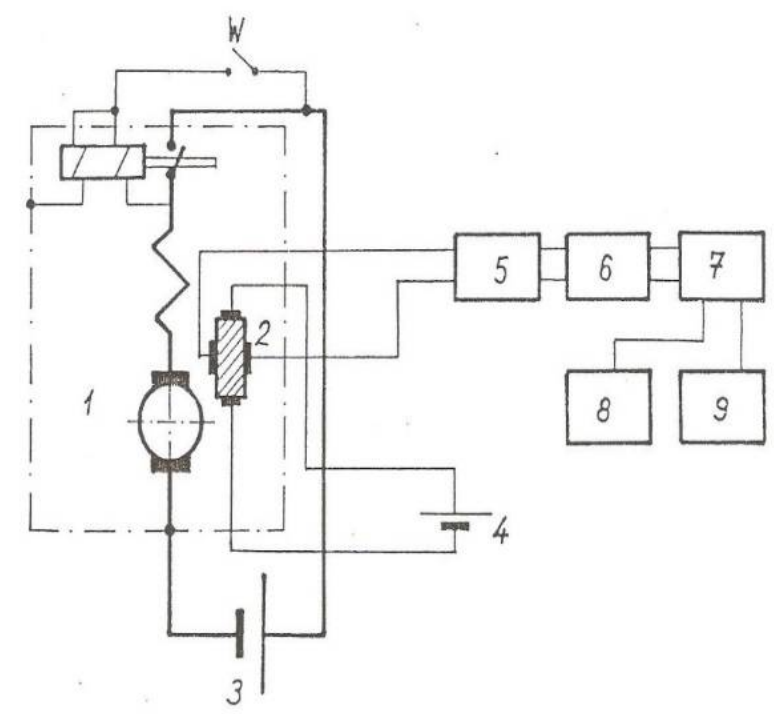

Figure 9. Block diagram of the stand: 1-starter, 2-Hall sensor, 3-battery, 4-Hall sensor supply, 5-signal amplifier, 6-protective system, 7-computer with an analogue-to-digital converter, 8-monitor, 9-printer.

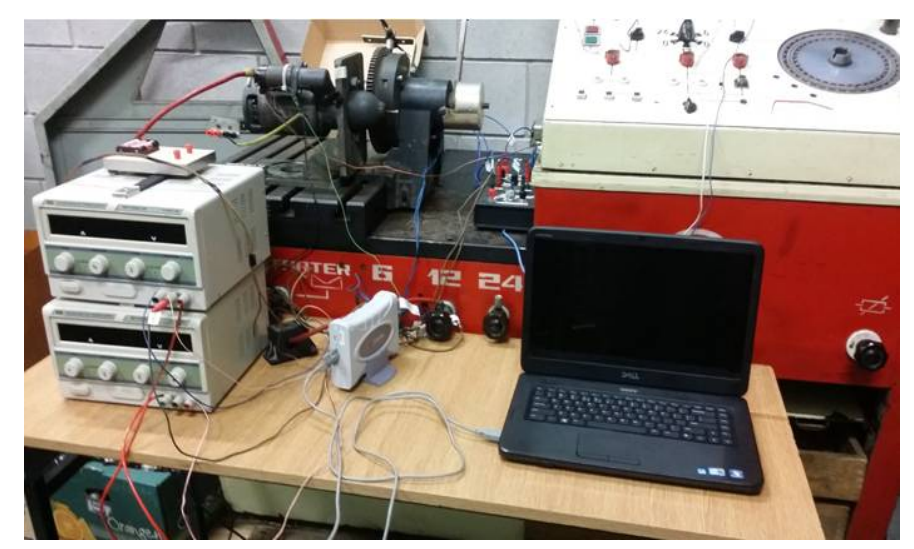

Figure 10. View of the measurement stand for testing the starter with the Hall sensor.

\subsection{The Course of Measurements}

Testing the starter with the Hall sensors consisted in recording the voltage waveforms obtained from the Hall sensors, which was commensurate with magnetic induction (magnetic flux B is proportional to Hall voltage and inversely proportional to the supply current with a constant Hall sensor coefficient). The Hall voltage signal was amplified on the voltage amplifier. 
As one Hall sensor was placed on the rotor and the other one on the stator, it can be assumed that both Hall sensors were in the air gap and thus recorded the course of induction in the gap. It turns out that such a course is different for different failures and differs from the course of an efficient starter. Induction distribution in the air gap can be a diagnostic parameter of the starter and can be used to assess its technical condition. The tests were carried out in such a way that the course of induction was recorded for the unloaded starter, i.e. at its idle running, and for the loaded one, but with the same load for all cases.

\section{Test Results}

The Hall voltage sequence for selected failures and changed time base ranges of the observed waveforms (to identify differences) is shown in Figures 11-18.

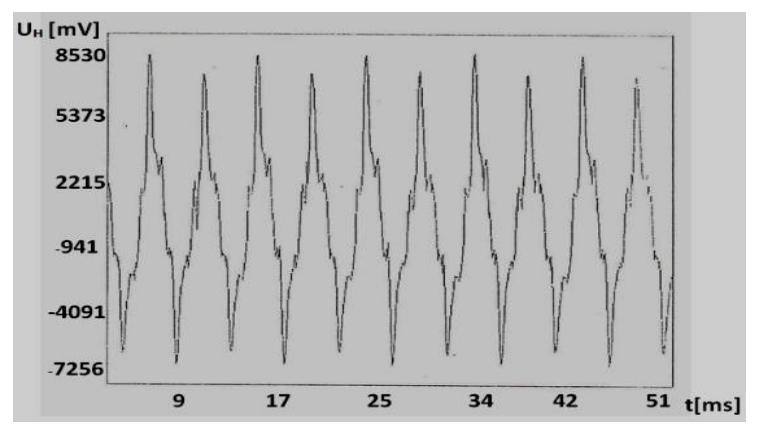

Figure 11. Hall voltage versus time for efficient starter, Hall sensors fixed on the rotor, unloaded starter.

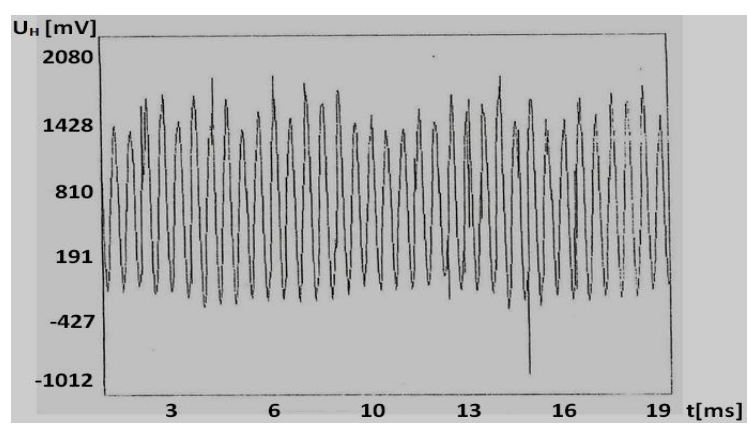

Figure 12. Hall voltage versus time for efficient starter, Hall sensors fixed on the stator, unloaded starter.

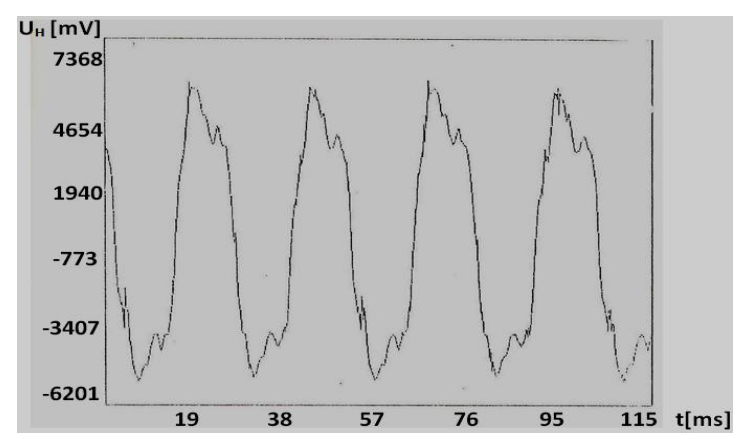

Figure 13. Hall voltage versus time in the case of the shorting of two commutator sectors, Hall sensors fixed on the rotor, loaded starter. 


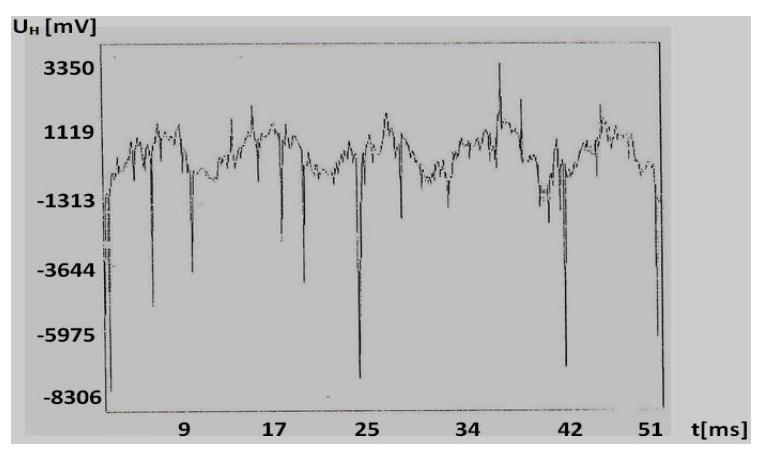

Figure 14. Hall voltage versus time in the case of the shorting of two commutator sectors, Hall sensors fixed on the stator, loaded starter.

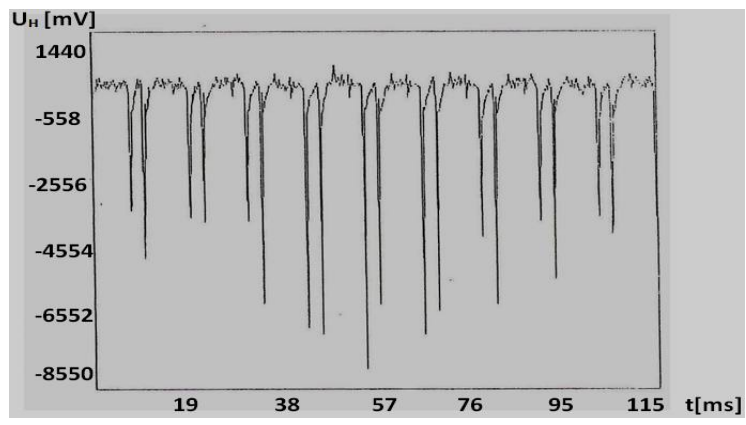

Figure 15. Hall voltage versus time in the case of breaking the rotor winding integrity, Hall sensors fixed on the rotor, unloaded starter.

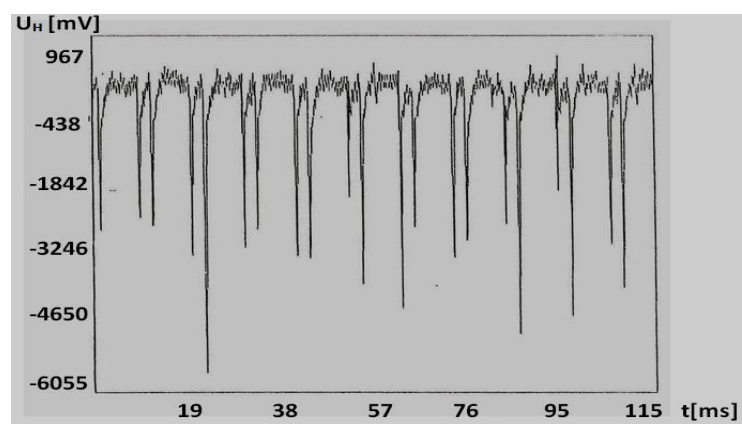

Figure 16. Hall voltage versus time in the case of breaking the rotor winding integrity, Hall sensors fixed on the stator, unloaded starter.

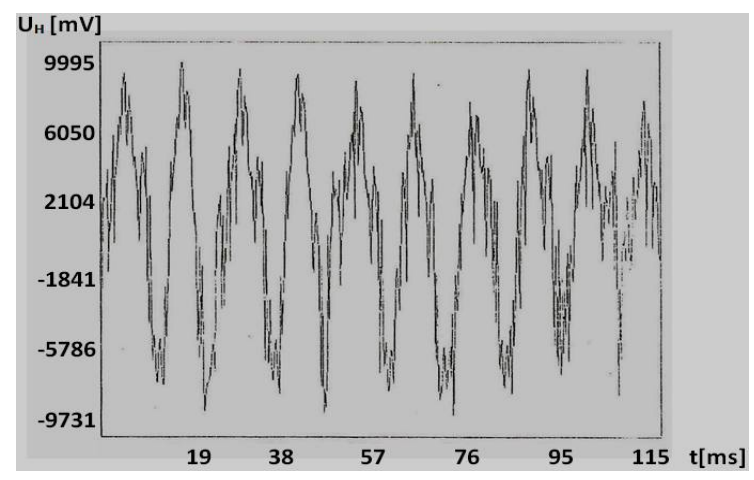

Figure 17. Hall voltage versus time in the case of brush wear, Hall sensors fixed on the rotor, unloaded starter. 


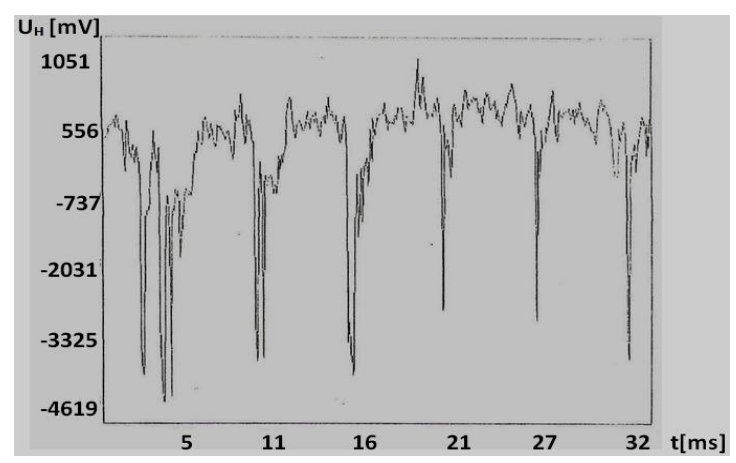

Figure 18. Hall voltage versus time in case of brush wear, Hall sensors fixed on the stator, loaded starter.

The waveforms of induction distribution measured by Hall sensors fixed on the rotor and on the stator are very similar. However, the course of induction recorded by the Hall sensor fixed on the rotor provides more information useful in the analysis of starter failures in the form of a short circuit and a break in the rotor windings, which is related to the curve of the rotor and stator interaction field (Equation (7)).

The waveforms of failures, in relation to the charts of the efficient starter, are more regular. e.g., for the shorting of two commutator sectors. Their course is characteristic in the case of breaks in the rotor winding. A completely different course is obtained here and its character does not resemble any of those discussed earlier. What makes it characteristic are the visible peaks going down, probably caused by power surges resulting from breaks in the current circuit of the starter at the moment when the last commutator sector (before the sector whose winding solder was removed) emerged from under the brush, breaking the starter induction circuit.

\section{Conclusions}

The tests of the starter carried out with the Hall sensor confirm the assumptions that it can be used for car starter diagnostics, and at the same time for assessing its technical condition. It can also be concluded from the tests that diagnosing starters by means of the Hall sensor placed on the pole shoe is more useful (because it is easy to draw the Hall sensor wires out).

Although the system with the Hall sensor placed on the rotor provides more information about magnetic field distribution in the armature gap, it is more complicated as it requires using additional slip-rings and brushes necessary for letting wires out of the Hall sensor placed on the movable rotor. Such an option can be used in laboratory tests. The recorded voltage waveforms allowed for the elaboration of a diagnostic model for an operational control system of the starter condition for motor transport. Characteristic changes in the course of registered magnetic induction were subjected to FFT analysis, and a database for individual failures was developed, which is the subject of other articles.

Author Contributions: All authors have contributed equally to this work. All authors were involved in the preparation of the manuscript.

Conflicts of Interest: The authors declare no conflict of interest.

\section{References}

1. $\quad$ Biteus, J.; Frisk, E.; Nyberg, M. Distributed Diagnosis Using a Condensed Representation of Diagnoses With Application to an Automotive Vehicle. IEEE Trans. Syst. Man Cybern. Part A Syst. Hum. 2011, 6, 1262-1267. [CrossRef]

2. Dziubiński, M.; Siemionek, E.; Drozd, A.; Jarzyna, W.; Kołodziej, S. Analysis of energy consumption of public transport in Lublin. In Proceedings of the 5th National Congress of Environmental Engineering, Lublin, Poland, 29 May-1 June 2016; pp. 293-298. 
3. Dziubiński, M. Ecological aspect of electronic ignition and electronic injection system. In Proceedings of the 5th National Congress of Environmental Engineering, Lublin, Poland, 29 May-1 June 2016; pp. 299-303.

4. Zaidi, S.; Aviyente, S.; Salman, M.; Shin, K. Prognosis of gear failures in DC starter motors using hidden Markov Models. IEEE Trans. Ind. Electron. 2011, 5, 1695-1706. [CrossRef]

5. Bay, O.; Bayir, R. A fault diagnosis of engine starting system via starter motors using fuzzy logic algorithm. Gazi J. Univ. Sci. 2011, 3, 437-440.

6. Murugesan, V.; Chandramohan, G.; Senthil, M.; Rudramoorthy, R.; Ashok, L.; Suresh, R.; Basha, D.; Vishnu, K. An overview of automobile starting system faults and fault diagnosis methods. ARPN J. Eng. Appl. Sci. 2012, 7, 812-819.

7. Pszczółkowski, J.; Trawiński, G. Engine crankshaft driving with the help of electric and pneumatic starter. Logistics 2011, 6, 3499-3508.

8. Svärd, C.; Nyberg, M. Residual Generators for Fault Diagnosis Using Computation Sequences with Mixed Causality Applied to Automotive Systems. IEEE Trans. Syst. Man Cybern. Part A Syst. Hum. 2010, 6, 1310-1328. [CrossRef]

9. Frisk, E.; Krysander, M.; Nyberg, M.; Aslund, J. A toolbox for design of diagnosis systems. In Proceedings of the 6th IFAC Symposium on Fault Detection, Supervision and Safety of Technical Processes International Federation of Automatic Control, Beijing, China, 30 August-1 September 2006.

10. Issermann, R. Model-based fault-detection and diagnosis-Status and applications. Ann. Rev. Control 2005, 1, 71-75. [CrossRef]

11. Nyberg, M.; Frisk, E. A derivation of the minimal polynominal basis approach to linear residual generation. J. Autom. 2001, 9, 1417-1424.

12. Patton, R.; Chen, J. Design methods for robust fault diagnosis. Control Syst. Robot. Autom. 2009, 16, 84-111.

13. Fuvesi, V.; Kovacs, E.; Blaga, C. Measurement and identification of a starter motor system. In Proceedings of the 2nd International Conference on Recent Achievements in Mechatronics, Automation, Computer science and Robotics (Macro), Cluj-Napoca, Romania, 14-15 May 2010; pp. 129-134.

14. Ausserlechner, U. A method to compute the Hall-geometry factor at weak magnetic field in closed analytical form. Electr. Eng. 2016, 3, 189-206. [CrossRef]

15. Dziubiński, M.; Drozd, A.; Adamiec, M.; Siemionek, E. Simulation tests of the starting system. Pozn. Univ. Technol. Acad. J. Electr. Eng. 2016, 88, 89-100.

16. Dziubiński, M.; Ocioszyński, J.; Walusiak, S. Electrical Engineering and Electronics of Automotive; Publishing House of the Lublin University of Technology: Lublin, Poland, 1999.

17. Dziubiński, M.; Plich, M.; Drozd, A. Modelling and simulation characteristics of starting system. J. Konbin 2016, 37, 277-296. [CrossRef]

18. Saidani, S.; Ghariani, M. Switched Reluctance Machine for A Sterter-Alternator Micro-Hybrid Car. Intell. Control Autom. 2015, 6, 271-288. [CrossRef]

19. Dabrowski, M. Issues optimal research design series of electrical machines. In Proceedings of the $\mathrm{XL}$ International Symposium on Electrical Machines, Hajnowka, Poland, 15-18 June 2004; pp. 39-44.

20. Wang, X.; Hahn, S.; Jung, H. Performance analysis and optialization of a permanent-magnet starter motor with auxiliary poles by FEM. IEEE Trans. Magn. 2003, 3, 1476-1479. [CrossRef]

(C) 2018 by the authors. Licensee MDPI, Basel, Switzerland. This article is an open access article distributed under the terms and conditions of the Creative Commons Attribution (CC BY) license (http://creativecommons.org/licenses/by/4.0/). 